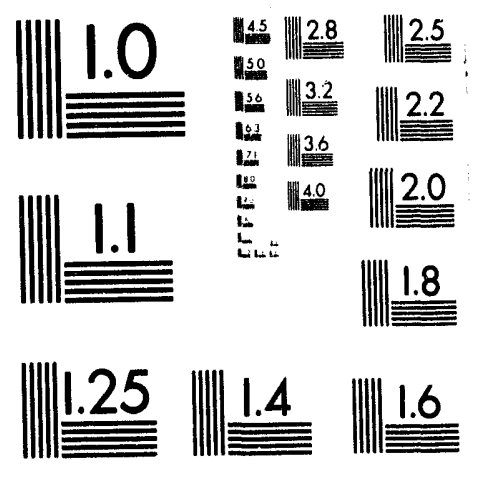



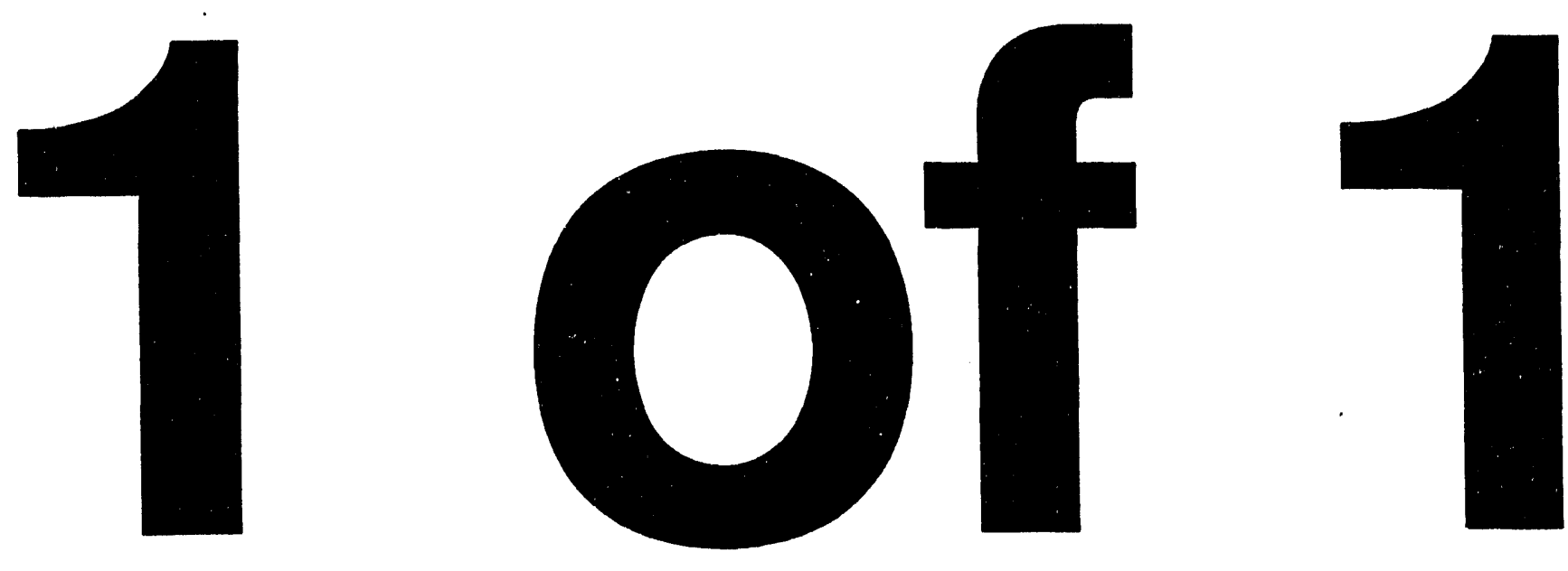


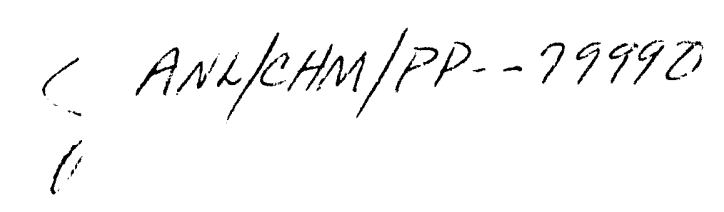

Theory for Electron-Transfer Reactions

in a Three-Component System: the "Degenerate" Regime

\section{Jau Tang}

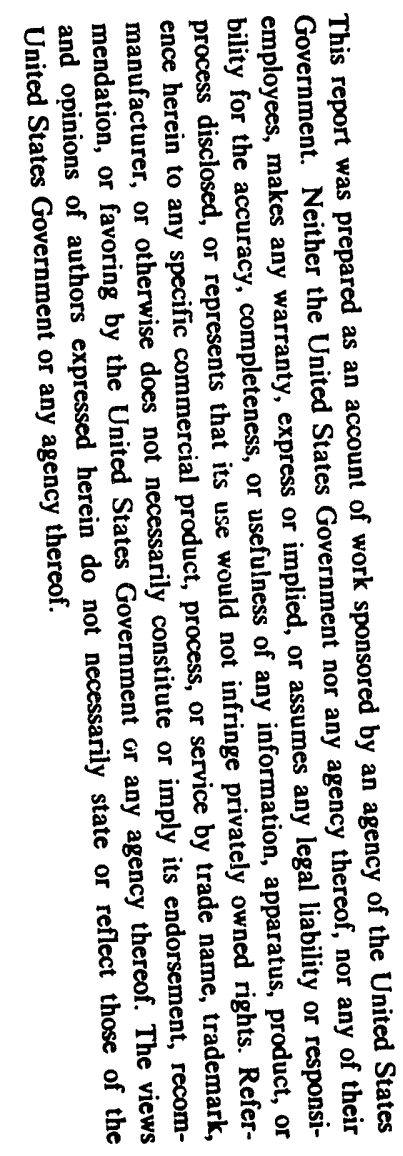

Chemistry Division, Argonne National Laboratory

Argonne, IL 60439 


\begin{abstract}

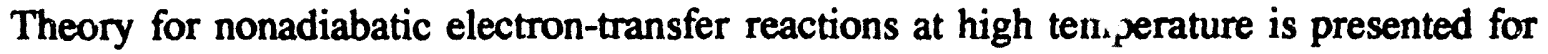
a three-component system (a donor, an acceptor, and a bridging intermediate). Only the "degenerate" regime of triple energy-level crossing is considered assuming three Marcus parabolas cross at the same point and the direct free energy gap vanishes. Because the ordinary second-order perturbation theory fails, the nonperturbative stochastic Liouville approach of Zusman is used in deriving analytical expressions for the superexchange and the sequential electron-transfer rate constants.
\end{abstract}




\section{Introduction}

Most of the theoretical treatments of electron-transfer reactions since the pioneering study of Marcus,' have primarily focused on a two-component system with a donor and an acceptor. More recently there are some attempts ${ }^{2-9}$ to extend the theory to a three-component system in which an intermediate bridges a donor with a distant acceptor. In the approach of Mukamel et al., ${ }^{5,6}$ the Padé approximation was assumed to include higher order terms of perturbation series for the degenerate case where the direct free energy gap vanishes and the second-order perturbation theory fails. In another simpler approach by Tang et al. ${ }^{7-9}$ the nonperturbative approach of Zusman ${ }^{10}$ is used. In the Zusman's stochastic Liouville approach, the solvent motion is assumed to be overdamped and follows the classical description of Smoluchowski diffusion. In the more recent work, ${ }^{9}$ an analytical rate expression for both superexchange and sequential processes was derived. The derived formula for both sequential and superexchange electrontransfer rate constants bridges between the nonadiabatic and solvent-controlled adiabatic regimes. However, only the "nondegenerate" regime was considered there, assuming the direct free energy gap is much larger than the electronic couplings. As an extension to the previous reports, we will focus in this work on the "degenerate" case where the direct vertical energy gap between the donor and the intermediate vanishes and the three Marcus parabolas cross at the same point.

\section{The stochastic Liouville theory and}

In this work we consider a three-component system involving a donor, an intermediate, and an acceptor, represented by $|1\rangle,|2\rangle$, and $|3\rangle$, respectively. Here we shall assume a large separation between the donor and the acceptor. Although there is no direct electronic 
coupling between them, they can interfere with each other in a superexchange manner through the intermediate state with the coupling to the donor $\left(\mathrm{V}_{12}\right)$ and to the acceptor $\left(\mathrm{V}_{23}\right)$. As shown in Fig. 1, each component is ass: zned a Marcus free-energy parabola $U_{k}(x)$ in the reaction coordinate

$$
\begin{aligned}
& U_{1}(x)=\frac{\lambda}{2} x^{2} \\
& U_{2}(x)=\frac{\lambda}{2}\left(x-x_{2}\right)^{2}-E_{g(12)} \\
& U_{3}(x)=\frac{\lambda}{2}\left(x-x_{3}\right)^{2}-E_{g(13)},
\end{aligned}
$$

where $\lambda$ is the force constant; $E_{g(12)}$ (or $E_{g(13)}$ ) is the free-energy gap defined as the energy separation between the bottoms of the second parabola (or the third) and the first one. The bottom of the parabola $U_{k}(x)$ is assumed at $x_{k}$, and $x_{1}$ is at the origin. The reorganization energy $E_{r(i)}$ can be related to these displacements $x_{i}$ and $x_{j}$ by

$$
E_{x(D)}=\frac{\lambda}{2}\left(x_{1}-x_{j}\right)^{2} .
$$

For the "degenerate" case treated here where all three parabolas cross at $\mathrm{x}=0$, one has $\mathrm{E}_{\mathrm{r}(\mathrm{i})}=$ $\mathrm{E}_{\mathrm{g}(i)}$. The Zusman's stochastic Liouville equation for a three-component system can be described in the following Laplace transform? with the variable $\mathrm{s}$ 


$$
\begin{aligned}
& s \hat{p}_{11}-\rho_{11}(0)=i \frac{V_{12}}{h}\left(\hat{p}_{12}-\hat{p}_{21}\right)+L_{1} \hat{p}_{11} \\
& s \hat{p}_{22}=-i \frac{V_{12}}{\hbar}\left(\hat{p}_{12}-\hat{p}_{21}\right)+i \frac{V_{23}}{\hbar}\left(\hat{\rho}_{23}-\hat{\rho}_{32}\right)+L_{2} \hat{p}_{22} \\
& s \hat{p}_{33}=-i \frac{V_{23}}{h}\left(\hat{p}_{23}-\hat{p}_{32}\right)+L_{3} \hat{p}_{33} \\
& s \hat{\rho}_{12}=-i \frac{U_{12}}{h} \hat{\rho}_{12}+i \frac{V_{12}}{h}\left(\hat{\rho}_{11}-\hat{\rho}_{22}\right)+i \frac{V_{23}}{h} \hat{p}_{13}+L_{12} \hat{\rho}_{12} \\
& s \hat{\rho}_{23}=-i \frac{U_{23}}{\hbar} \hat{\boldsymbol{p}}_{23}+i \frac{V_{23}}{\hbar}\left(\hat{\boldsymbol{p}}_{22}-\hat{\rho}_{33}\right)-i \frac{V_{12}}{\hbar} \hat{\boldsymbol{p}}_{13}+L_{23} \hat{\boldsymbol{\rho}}_{23} \\
& S \hat{p}_{13}=-i \frac{U_{13}}{\hbar} \hat{\rho}_{13}-i \frac{V_{12}}{h} \hat{p}_{23}+i \frac{V_{23}}{\hbar} \hat{\rho}_{12}+L_{13} \hat{p}_{13} \\
& \hat{\boldsymbol{p}}_{21}=\hat{\boldsymbol{p}}_{12}^{*} \\
& \hat{\boldsymbol{\rho}}_{32}=\hat{\boldsymbol{\rho}}_{23}^{*} \\
& \hat{\mathbf{p}}_{31}=\hat{\boldsymbol{p}}_{13}^{*},
\end{aligned}
$$

The donor and the acceptor are assumed to be separated by a great distance and thus $V_{13}$ vanishes. Here $U_{i j}(x)$ is defined as $U_{i}(x)-U_{j}(x)$. The Smoluchowski diffusion operator $L_{i}$ is given by

$$
L_{1}=\frac{1}{\tau_{L}}\left[1+\left(x-x_{1}\right) \frac{\partial}{\partial x}\right]+\frac{k_{B} T}{\lambda \tau_{L}} \frac{\partial^{2}}{\partial x^{2}}
$$

where $\tau_{\mathrm{L}}$ known as the longitudinal relaxation time is a constant for a Debye solvent. The diffusion operator $L_{i j}$ for the off-diagonal elements $\hat{\rho}_{12}, \hat{\rho}_{23}$, and $\hat{\rho}_{13}$ of the density matrix can be 
neglected because of fast oscillation of these off-diagonal density-matrix elements. ${ }^{10,11}$ The subset of eq. (3) involving these off-diagonal elements can be given by

$$
\left(\begin{array}{ccc}
s+\frac{i}{\hbar} U_{12}(x) & 0 & -\frac{i}{\hbar} V_{23} \\
0 & s+\frac{i}{\hbar} U_{23}(x) & \frac{i}{\hbar} V_{12} \\
-\frac{i}{\hbar} V_{23} & \frac{i}{\hbar} V_{12} & s+\frac{i}{\hbar} U_{13}(x)
\end{array}\right)\left(\begin{array}{c}
\hat{\rho}_{12} \\
\hat{\rho}_{23} \\
\hat{\rho}_{13}
\end{array}\right)=\left(\begin{array}{c}
\frac{i}{\hbar} V_{12}\left(\hat{\rho}_{11}-\hat{\rho}_{22}\right) \\
\frac{i}{\hbar} V_{23}\left(\hat{\rho}_{22}-\hat{\rho}_{33}\right) \\
0
\end{array}\right)
$$

One can solve eq. (5) for $\hat{\rho}_{12}$ and $\hat{\rho}_{23}$ which are given by

$$
\begin{aligned}
& \hat{p}_{12}=i \frac{V_{12}}{\hbar} \frac{\left(\hat{p}_{11}-\hat{p}_{22}\right)\left[\left(s+i \frac{U_{13}}{\hbar}\right)\left(s+i \frac{U_{23}}{\hbar}\right)+\frac{V_{12}^{2}}{\hbar^{2}}\right]+\left(\hat{p}_{22}-\hat{p}_{33}\right) \frac{V_{23}^{2}}{\hbar^{2}}}{D(s)} \\
& \hat{\rho}_{23}=i \frac{V_{23}}{\hbar} \frac{\left(\hat{p}_{22}-\hat{p}_{33}\right)\left[\left(s+i \frac{U_{13}}{\hbar}\right)\left(s+i \frac{U_{12}}{\hbar}\right)+\frac{V_{23}^{2}}{\hbar^{2}}\right]+\left(\hat{p}_{11}-\hat{p}_{22}\right) \frac{V_{12}^{2}}{\hbar^{2}}}{D(s)} \\
& D(s)=\left(s+i \frac{U_{12}}{\hbar}\right)\left(s+i \frac{U_{23}}{h}\right)\left(s+i \frac{U_{13}}{\hbar}\right)+\frac{V_{12}^{2}}{\hbar^{2}}\left(s+i \frac{U_{12}}{\hbar}\right)+\frac{V_{23}^{2}}{\hbar^{2}}\left(s+i \frac{U_{23}}{\hbar}\right) .
\end{aligned}
$$

From eq. (1) one can show $U_{i j}(x)=x / \xi_{i j}$ where $\xi_{i j}=1 /\left(2 \lambda E_{r i j i j}\right)^{1 / 2}$. The roots for the determinant of the matrix in eq. (5), $D(s)$, can be solved by assuming a form of $-i \alpha_{1} x / \hbar+\alpha_{2}$ for the root, and then determining $\alpha_{1}$ and $\alpha_{2}$ by their substitution in $\mathrm{D}(\mathrm{s})$ while retaining the lowest order terms of $x$ in the expansion. Three roots $s_{1}, s_{2}$ and $s_{3}$ can be found accordingly and they are given by 


$$
\begin{aligned}
& s_{1}=-i \frac{x}{\hbar \xi_{0}} \\
& s_{2,3}=-i \frac{x}{h \xi_{1}} \pm i \omega_{1} \\
& \frac{1}{\xi_{0}}=\frac{v_{12}^{2} / \xi_{12}+V_{23}^{2} / \xi_{23}}{V_{12}^{2}+V_{23}^{2}} \\
& \frac{1}{\xi_{3}}=\frac{1}{2}\left(\frac{1}{\xi_{12}}+\frac{1}{\xi_{23}}+\frac{1}{\xi_{13}}-\frac{1}{\xi_{0}}\right) \\
& \omega_{1}=\frac{1}{\hbar} \sqrt{V_{12}^{2}+V_{23}^{2}}
\end{aligned}
$$

One can expand $\hat{\rho}_{12}$ and $\hat{\rho}_{23}$ in eq. (6) about their poles which are the roots of $D(s) g$ iven in the above equation

$$
\begin{aligned}
& \hat{\rho}_{12}=i \frac{V_{12}}{\hbar}\left[\left(\hat{\rho}_{11}-\hat{\rho}_{2}\right) \frac{V_{12}^{2}}{\hbar^{2} \omega_{8}^{2}}+\left(\hat{\rho}_{22}-\hat{\rho}_{33}\right) \frac{V_{23}^{2}}{\hbar^{2} \omega_{1}^{2}}\right] \frac{1}{s+i \frac{x}{\hbar \xi_{0}}} \\
& +i \frac{V_{12}}{\hbar}\left(\hat{p}_{11}+\hat{\rho}_{33}-2 \hat{\rho}_{25}\right) \frac{V_{23}^{2}}{\hbar^{2} \omega_{s}^{2}} \frac{1}{2}\left[\frac{1}{s+i \frac{x-x_{s}}{\hbar \xi_{g}}}+\frac{1}{s+i \frac{x+x_{s}}{\hbar \xi_{g}}}\right] \\
& \hat{\rho}_{23}=i \frac{V_{23}}{\hbar}\left[\left(\hat{\rho}_{11}-\hat{\rho}_{22}\right) \frac{V_{12}^{2}}{\hbar^{2} \omega_{1}^{2}}+\left(\hat{\rho}_{22}-\hat{\rho}_{33}\right) \frac{V_{23}^{2}}{\hbar^{2} \omega_{1}^{2}}\right] \frac{1}{s+i \frac{x}{\hbar \xi_{0}}} \\
& -i \frac{V_{23}}{\hbar}\left(\hat{\rho}_{11}+\hat{\rho}_{33}-2 \hat{\rho}_{20}\right) \frac{V_{12}^{2}}{\hbar^{2} \omega_{a}^{2}} \frac{1}{2}\left[\frac{1}{s+i \frac{x-x_{a}}{\hbar \xi_{a}}}+\frac{1}{s+i \frac{x+x_{8}}{\hbar \xi_{g}}}\right] \text {. }
\end{aligned}
$$


where $x_{i}=\hbar \omega_{b} \xi_{2}$. The above equation indicates that although these parabolas $U_{1}(x), U_{2}(x)$ and $\mathrm{U}_{3}(\mathrm{x})$ cross at $\mathrm{x}=0$, however, because of their interactions, the actual effective crossing points are now at $x=0,-x_{1}$ and $x_{2}$. By combining the above equation with eq. (3) and with the approximation of $2 \mathrm{~s} /\left(\hbar^{2} \mathrm{~s}^{2}+\mathrm{x}^{2} / \xi_{0}{ }^{2}\right)$ by a $\delta$-function $2 \pi \xi_{0} \delta(\mathrm{x}) / \hbar$ and $2 \mathrm{~s} /\left(\boldsymbol{h}^{2} \mathrm{~s}^{2}+\left(\mathrm{x} \pm \mathrm{x}_{\mathrm{s}}\right)^{2} / \xi_{\mathrm{s}}{ }^{2}\right)$ by $2 \pi \xi_{s} \delta\left(x \pm x_{s}\right) / h$, one can show

$s \hat{\rho}_{11}-\rho_{11}(0)=-\frac{2 \pi}{\hbar} \delta(x) \xi_{0}\left[W_{12}^{2}\left(\hat{\rho}_{11}-\hat{\rho}_{22}\right)+W_{s}^{2}\left(\hat{\rho}_{22}-\hat{\rho}_{33}\right)\right]$

$$
-\frac{2 \pi}{h}\left[\delta\left(x-x_{g}\right)+\delta\left(x+x_{g}\right)\right] \frac{\xi_{s}}{2} W_{s}^{2}\left(\hat{\rho}_{11}+\hat{\rho}_{33}-2 \hat{\rho}_{22}\right)+L_{1} \hat{\rho}_{11}
$$

$s \hat{\rho}_{22}=\frac{2 \pi}{\hbar} \delta(x) \xi_{0}\left[W_{12}^{2}\left(\hat{\rho}_{11}-\hat{\rho}_{22}\right)+W_{s}^{2}\left(\hat{\rho}_{22}-\hat{\rho}_{33}\right)\right]$

$$
\begin{aligned}
& -\frac{2 \pi}{\hbar} \delta(x) \xi_{0}\left[W_{s}^{2}\left(\hat{\rho}_{11}-\hat{\rho}_{22}\right)+W_{23}^{2}\left(\hat{\rho}_{22}-\hat{\rho}_{33}\right)\right] \\
& +\frac{2 \pi}{\hbar}\left[\delta\left(x-x_{8}\right)+\delta\left(x+x_{8}\right)\right] \xi_{8} W_{s}^{2}\left(\hat{\rho}_{11}+\hat{\rho}_{33}-2 \hat{\rho}_{22}\right)+L_{2} \hat{\rho}_{22}
\end{aligned}
$$

s $\hat{\rho}_{33}=\frac{2 \pi}{\hbar} \delta(x) \xi_{0}\left[W_{s}^{2}\left(\hat{\rho}_{11}-\hat{\rho}_{22}\right)+W_{23}^{2}\left(\hat{\rho}_{22}-\hat{\rho}_{33}\right)\right]$

$$
-\frac{2 \pi}{\hbar}\left[8\left(x-x_{8}\right)+8\left(x+x_{8}\right)\right] \frac{\xi_{s}}{2} W_{s}^{2}\left(\hat{\rho}_{11}+\hat{\rho}_{33}-2 \hat{\rho}_{22}\right)+L_{3} \hat{\rho}_{33},
$$

where we have used the following notation for the effective coupling constants: 


$$
\begin{aligned}
& \mathrm{W}_{12}=\frac{\mathrm{V}_{12}^{2}}{\sqrt{\mathrm{V}_{12}^{2}+\mathrm{V}_{23}^{2}}} \\
& \mathrm{~W}_{23}=\frac{\mathrm{V}_{23}^{2}}{\sqrt{\mathrm{V}_{12}^{2}+\mathrm{V}_{23}^{2}}} \\
& \mathrm{~W}_{\mathrm{s}}=\frac{\mathrm{V}_{12} \mathrm{~V}_{23}}{\sqrt{\mathrm{V}_{12}^{2}+\mathrm{V}_{23}^{2}}} .
\end{aligned}
$$

In the "nondegenerate" case with a large direct free energy gap, the sequential and the superexchange reactions occur through a different pathway, i.e., the transition occurs at a different crossing point for a different mechanism. ${ }^{9}$ However, in this "degenerate" case, both the sequential and the superexchange transition can occur at all three effective crossing points $0,-x_{8}$ and $x_{8}$.

Eq. (9) can be solved by the Green's function method.9.11 The Laplace transform of the Green's function satisfies the following Smoluchowski equation

$$
\left(s-L_{k}\right) \hat{G}_{k}\left(x, x^{\prime}\right)=8\left(x-x^{\prime}\right), \quad k=1,2,3 \text {. }
$$

One can express eq. (9) in terms of the Green's function 


$$
\begin{aligned}
& \hat{\rho}_{11}(x)=\frac{1}{s} \rho_{11,0 q}(x)-\frac{2 \pi}{\hbar} \hat{G}_{1}(x, 0) \xi_{0}\left[W_{12}^{2}\left(\hat{\rho}_{11}(0)-\hat{\rho}_{22}(0)\right)+W_{s}^{2}\left(\hat{\rho}_{22}(0)-\hat{\rho}_{33}(0)\right)\right] \\
& -\frac{2 \pi}{\hbar} \hat{G}_{1}\left(x_{,}-x_{8}\right) \frac{\xi_{s}}{2} W_{S}^{2}\left(\hat{\rho}_{11}\left(-x_{g}\right)+\hat{\rho}_{33}\left(-x_{8}\right)-2 \hat{\rho}_{22}\left(-x_{g}\right)\right) \\
& -\frac{2 \pi}{\hbar} \hat{G}_{1}\left(x, x_{8}\right) \frac{\xi_{s}}{2} W_{s}^{2}\left(\hat{\rho}_{11}\left(x_{8}\right)+\hat{\rho}_{33}\left(x_{8}\right)-2 \hat{\rho}_{22}\left(x_{8}\right)\right) \\
& \hat{\rho}_{22}(x)=\frac{2 \pi}{\hbar} \hat{G}_{2}(x, 0) \xi_{0}\left[W_{12}^{2}\left(\hat{\rho}_{11}(0)-\hat{\rho}_{22}(0)\right)+W_{s}^{2}\left(\hat{\rho}_{22}(0)-\hat{\rho}_{33}(0)\right)\right] \\
& -\frac{2 \pi}{\hbar} \hat{G}_{2}(\mathrm{x}, 0) \xi_{0}\left[W_{3}^{2}\left(\hat{\rho}_{11}(0)-\hat{\rho}_{22}(0)\right)+W_{23}^{2}\left(\hat{\rho}_{22}(0)-\hat{\rho}_{33}(0)\right)\right] \\
& +\frac{2 \pi}{\hbar} \hat{G}_{2}\left(x_{,}-x_{8}\right) \xi_{s} W_{s}^{2}\left(\hat{\rho}_{11}\left(-x_{8}\right)+\hat{\rho}_{33}\left(-x_{8}\right)-2 \hat{\rho}_{22}\left(-x_{8}\right)\right) \\
& +\frac{2 \pi}{\hbar} \hat{G}_{2}\left(x_{8} x_{8}\right) \xi_{8} W_{8}^{2}\left(\hat{p}_{11}\left(x_{8}\right)+\hat{\rho}_{33}\left(x_{8}\right)-2 \hat{\rho}_{22}\left(x_{8}\right)\right) \\
& \hat{\rho}_{33}(x)=\frac{2 \pi}{\hbar} \hat{G}_{3}(x, 0) \xi_{0}\left[W_{s}^{2}\left(\hat{\rho}_{11}(0)-\hat{\rho}_{22}(0)\right)+W_{23}^{2}\left(\hat{\rho}_{22}(0)-\hat{\rho}_{33}(0)\right)\right] \\
& -\frac{2 \pi}{\hbar} \hat{G}_{3}\left(x_{,}-x_{8}\right) \frac{\xi_{8}}{2} W_{s}^{2}\left(\hat{\rho}_{11}\left(-x_{8}\right)+\hat{\rho}_{33}\left(-x_{8}\right)-2 \hat{\rho}_{22}\left(-x_{8}\right)\right) \\
& -\frac{2 \pi}{\hbar} \hat{G}_{3}\left(x, x_{8}\right) \frac{\xi_{8}}{2} W_{s}^{2}\left(\hat{\rho}_{11}\left(x_{8}\right)+\hat{\rho}_{33}\left(x_{8}\right)-2 \hat{\rho}_{22}\left(x_{8}\right)\right) .
\end{aligned}
$$

We shall now define $\hat{\sigma}_{1}(s), \hat{\sigma}_{2}(s)$ and $\hat{\sigma}_{3}(s)$ as the populations on each surface, i.e., $\int \mathrm{dx} \hat{\rho}_{\mathrm{kzk}}(\mathrm{x})$ (for $k=1,2,3$ ). By integrating $\hat{\rho}_{k k}(x)$ in eq. (12) over $x$, one can show 


$$
\begin{aligned}
& s \hat{\sigma}_{1}(s)-1=-\frac{2 \pi}{\hbar} \xi_{0}\left[W_{12}^{2}\left(\hat{\rho}_{11}(0)-\hat{\rho}_{22}(0)\right)+W_{s}^{2}\left(\hat{\rho}_{22}(0)-\hat{\rho}_{33}(0)\right)\right] \\
& -\frac{2 \pi}{h} \frac{\xi_{8}}{2} W_{s}^{2}\left(\hat{\rho}_{11}\left(-x_{8}\right)+\hat{\rho}_{33}\left(-x_{8}\right)-2 \hat{\rho}_{22}\left(-x_{8}\right)\right) \\
& -\frac{2 \pi}{h} \frac{\xi_{8}}{2} W_{s}^{2}\left(\hat{p}_{11}\left(x_{8}\right)+\hat{p}_{33}\left(x_{8}\right)-2 \hat{\rho}_{22}\left(x_{8}\right)\right) \\
& s \hat{\sigma}_{22}(s)=\frac{2 \pi}{\hbar} \xi_{0}\left[W_{12}^{2}\left(\hat{\rho}_{11}(0)-\hat{\rho}_{22}(0)\right)+W_{s}^{2}\left(\hat{\rho}_{22}(0)-\hat{\rho}_{33}(0)\right)\right] \\
& -\frac{2 \pi}{\hbar} \xi_{0}\left[W_{s}^{2}\left(\hat{\rho}_{11}(0)-\hat{\rho}_{22}(0)\right)+W_{23}^{2}\left(\hat{p}_{22}(0)-\hat{\rho}_{33}(0)\right)\right] \\
& +\frac{2 \pi}{\hbar} \xi_{8} W_{s}^{2}\left(\hat{\rho}_{11}\left(-x_{8}\right)+\hat{\rho}_{33}\left(-x_{8}\right)-2 \hat{\rho}_{22}\left(-x_{8}\right)\right) \\
& +\frac{2 \pi}{\hbar} \xi_{8} W_{s}^{2}\left(\hat{\rho}_{11}\left(x_{8}\right)+\hat{\rho}_{33}\left(x_{8}\right)-2 \hat{\rho}_{22}\left(x_{8}\right)\right) \\
& s \hat{\sigma}_{33}(s)=\frac{2 \pi}{\hbar} \xi_{0}\left[W_{s}^{2}\left(\hat{\rho}_{11}(0)-\hat{\rho}_{22}(0)\right)+W_{23}^{2}\left(\hat{\rho}_{22}(0)-\hat{\rho}_{33}(0)\right)\right] \\
& -\frac{2 \pi}{\hbar} \frac{\xi_{8}}{2} W_{s}^{2}\left(\hat{\rho}_{11}\left(-x_{8}\right)+\hat{\rho}_{33}\left(-x_{8}\right)-2 \hat{\rho}_{22}\left(-x_{8}\right)\right) \\
& -\frac{2 \pi}{\hbar}-\frac{\xi_{8}}{2} W_{s}^{2}\left(\hat{\rho}_{11}\left(x_{8}\right)+\hat{\rho}_{33}\left(x_{8}\right)-2 \hat{\rho}_{22}\left(x_{8}\right)\right) \text {. }
\end{aligned}
$$

For nonadiabatic process, $G_{k}\left(x, x^{\prime}\right)$ can be approximated by $\rho_{\mathrm{kk}, \text {,q }}(x) / \mathrm{s} .{ }^{11}$ With this approximation of $G_{k}\left(x, x^{\prime}\right)$ substituted in eq. (12), one can evaluate $\hat{\rho}_{k k}(x)$ at the crossing point $x_{c}$. It can be shown from eqs. (12) and (13) that $\hat{\rho}_{k t}\left(x_{c}\right)=\rho_{k x, e q}\left(x_{c}\right) \hat{\sigma}_{k}(s)$, where $x_{c}=0,-x_{c}$ or $x_{s}$. One may transform eq. (13) into the following kinetic equation involving only $\hat{\sigma}_{\mathrm{k}}(\mathrm{s})$ if one replaces $\hat{\rho}_{\mathrm{tk}}\left(\mathrm{x}_{\mathrm{c}}\right)$ in eq. (13) with $\rho_{\text {tteq }}\left(x_{c}\right) \hat{\sigma}_{k}(s)$ 


$$
\begin{aligned}
& s \hat{\sigma}_{1}(s)-1=-\left(k_{12}+k_{13}\right) \hat{\sigma}_{1}(s)+k_{21} \hat{\sigma}_{2}(s)+k_{31} \hat{\sigma}_{3}(s) \\
& s \hat{\sigma}_{2}(s)=k_{12} \hat{\sigma}_{1}(s)-\left(k_{21}+k_{23}\right) \hat{\sigma}_{2}(s)+k_{32} \hat{\sigma}_{3}(s) \\
& s \hat{\sigma}_{3}(s)=k_{13} \hat{\sigma}_{1}(s)+k_{23} \hat{\sigma}_{2}(s)-\left(k_{31}+k_{32}\right) \hat{\sigma}_{3}(s) .
\end{aligned}
$$

The sequential $\left(k_{12}, k_{21}, k_{23}, k_{92}\right)$ and the superexchange $\left(k_{13}, k_{11}\right)$ rate constants in the above equation are defined as

$$
\begin{aligned}
& k_{12}=\frac{2 \pi}{h}\left(W_{12}^{2}-W_{s}^{2}\right) \xi_{0} p_{11, \infty 9}(0)+\frac{2 \pi}{h} W_{s}^{2} \xi_{1}\left[p_{11, \infty q}\left(x_{d}\right)+p_{11,0 q}\left(-x_{8}\right)\right] \\
& k_{23}=\frac{2 \pi}{h}\left(W_{23}^{2}-W_{S}^{2}\right) \xi_{0} \rho_{22,09}(0)+\frac{2 \pi}{h} W_{S}^{2} \xi_{1}\left[\rho_{22,0 q}\left(x_{p}\right)+p_{22_{, 0 Q}}\left(-x_{s}\right)\right]
\end{aligned}
$$

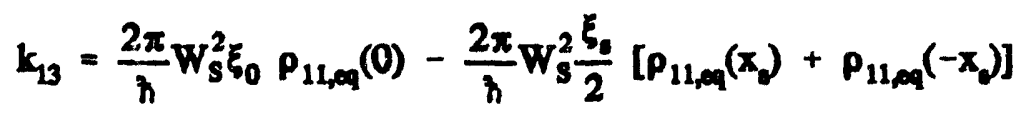

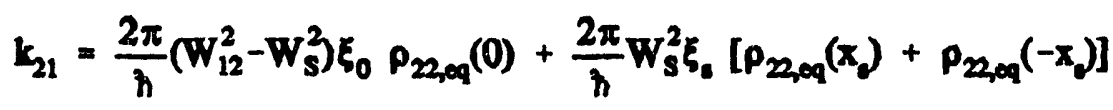

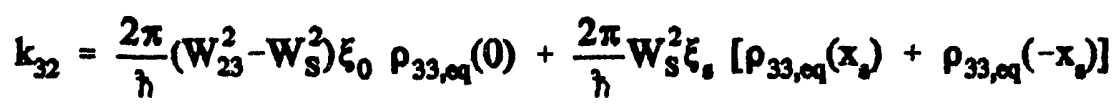

$$
\begin{aligned}
& k_{31}=\frac{2 \pi}{h} W_{s}^{2} \xi_{0} p_{33,09}(0)-\frac{2 \pi}{h} W_{s}^{2} \frac{\xi_{0}}{2}\left[p_{33, \infty q}\left(x_{0}\right)+p_{33_{, 09}}\left(-x_{d}\right)\right] .
\end{aligned}
$$

The Boltzmann distribution $\rho_{k k, o q}\left(x_{c}\right)$ at the crossing $x_{c}$ on the $k$-th surface is given by $\left(\lambda / 2 \pi k_{B} T\right)^{1 / 2} \exp \left(-\lambda\left(\pi_{c}-x_{k}\right)^{2} / 2 k_{B} T\right)$. The kinetic equation we have derived here describes nonadiabatic electron transfer among the states $|1\rangle,|2\rangle$, and $|3\rangle$ with the sequential transition rate constants given by $k_{12}, k_{23}$ and the superexchange transition rate constant given by 
$k_{13}$. It can be shown that the forward and the backward transition rate constants, $k_{i j}$ and $k_{j i}$, in eq. (15) satisfy the detailed balance principle. By substitution of $\xi_{0}, \xi_{3}, x_{3}$ and $W_{i j}$ as defined earlier, these rate constants in eq. (15) can be expressed in terms of $V_{12}, V_{23}$ and the reorganization energy $E_{\text {rid }}$. In particular, one can show

$$
\begin{aligned}
k_{13}=\frac{2 \pi}{h} \frac{1}{\sqrt{4 \pi k_{B} T}} \frac{V_{12}^{2} V_{23}^{2}}{V_{12}^{2}+V_{23}^{2}}\left[\frac{V_{12}^{2}+V_{23}^{2}}{V_{12}^{2} \sqrt{E_{r(12)}}+V_{23}^{2} \sqrt{E_{r(23)}}}\right. \\
-\frac{2 \exp \left[-\frac{V_{12}^{2}+V_{23}^{2}}{k_{B} T\left(\sqrt{E_{r(12)}}+\sqrt{E_{r(23)}}+\sqrt{E_{r(13)}}-\left(V_{12}^{2} \sqrt{E_{r(12)}}+V_{23}^{2} \sqrt{E_{r(23)}}\right) /\left(V_{12}^{2}+V_{23}^{2}\right)\right)}\right]}{\sqrt{E_{r(12)}}+\sqrt{E_{r(23)}}+\sqrt{E_{r(13)}}-\left(V_{12}^{2} \sqrt{E_{r(12)}}+V_{23}^{2} \sqrt{\left.E_{r(23)}\right)} /\left(V_{12}^{2}+V_{23}^{2}\right)\right.} .
\end{aligned}
$$

The second term in the above equation can be neglected if the couplings $V_{12}$ and $V_{23}$ are so strong that the exponent is much larger than one. On the other hand, for weak coupling three effective crossing points are very close and $\rho_{\mathrm{lt}, \mathrm{eq}}\left( \pm \mathrm{x}_{\mathrm{l}}\right)$ in eq. (15) can be approximated by $\rho_{k k, \infty q}(0)$. In this limit one has the following simple relation

$$
\begin{aligned}
& k_{12}=\frac{2 \pi}{\hbar}\left[W_{12}^{2} \xi_{0}+W_{s}^{2}\left(2 \xi_{8}-\xi_{0}\right)\right] \rho_{11,09}(0) \\
& k_{23}=\frac{2 \pi}{\hbar}\left[W_{23}^{2} \xi_{0}+W_{s}^{2}\left(2 \xi_{8}-\xi_{0}\right)\right] \rho_{22,09}(0) \\
& k_{13}=\frac{2 \pi}{\hbar} W_{s}^{2}\left(\xi_{0}-\xi_{9}\right) \rho_{11,0 q}(0) .
\end{aligned}
$$




\section{Conclusions}

We have applied the stochastic Liouville equation of Zusman to investigate the electrontransfer reactions for a three-component system involving a donor, a distant acceptor and a bridging intermediate. We have focused here the "degenerate" regime where the direct vertical energy gap vanishes and three Marcus parabolas cross at the same point. Although the ordinary second-order perturbation theory is invalid in this case, the non-perturbative approach of Zusman allows us to derive analytical expressions for the rate constants for both the sequential and the superexchange pathways. In this report, only the nonadiabatic limit is considered. Extension of this work to the more complicate adiabatic limit and the "quasi-degenerate" regime, where the direct free energy gap and the coupling strength are of the same order of magnitude, is now under investigation and will be reported elsewhere.

\section{Acknowledgement}

This work was supported by the U.S. Department of Energy, Office of Basic Energy Sciences, Division of Chemical Sciences, under Contract W-31-109-Eng-38. 


\section{Figure Captions}

Fig. 1. Schematic representation of the Marcus free-energy profiles in the reaction coordinate for the donor $\left(U_{1}\right)$, the intermediate $\left(U_{2}\right)$, and the acceptor $\left(U_{3}\right)$ in a three-component system. $\mathrm{E}_{\mathrm{g(12)}}$ and $\mathrm{E}_{\mathrm{g}(13)}$ are the corresponding free-energy gaps. In this "degenerate" regime, all three parabolas are assumed to cross at $x=0$. 


\section{References}

${ }^{1}$ R. A. Marcus, J. Chem. Phys. 24, 966 (1956); 24, 979 (1956).

${ }^{2}$ R. A. Marcus , Chem. Phys. Lett. 133, 471 (1987).

${ }^{3}$ M. Bixon and J. Jortner, J. Phys. Chem. 92, 7148 (1988).

${ }^{4}$ J. S. Joseph, W. Bruno and W. Bialek, J. Phys. Chem. 95, 6242 (1991).

5 Y. Hu and S. Mukamel, J. Chem. Phys. 91, 6973 (1989).

${ }^{6}$ Y. Hu and S. Mukamel Perspectives in Photosynthesis, edited by J. Jortner and B. Pullman (Kluwer Academic Publishers, The Netherlands, 1989).

7 J. Tang, Z.Wang, and J. R. Norris, J. Chem. Phys. (in press).

8 J. Tang and J. R. Norris, Chem. Phys. (submitted).

9 J. Tang, J. Chem. Phys. (submitted).

10 L. D. Zusman, Chem. Phys. 49, 295 (1980).

${ }^{11}$ I. Rips and J. Jortner, J. Chem. Phys. 87, 6513 (1987). 


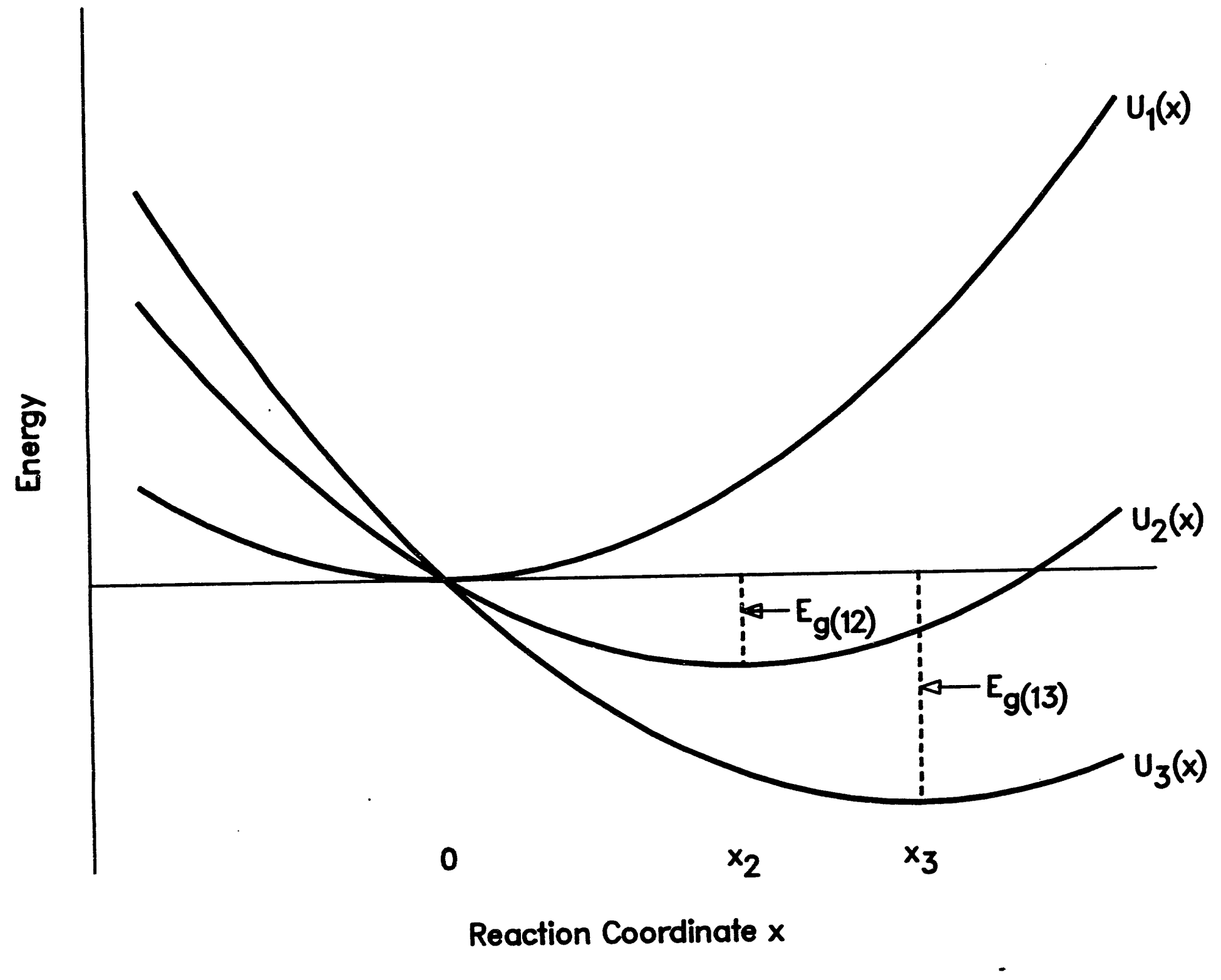



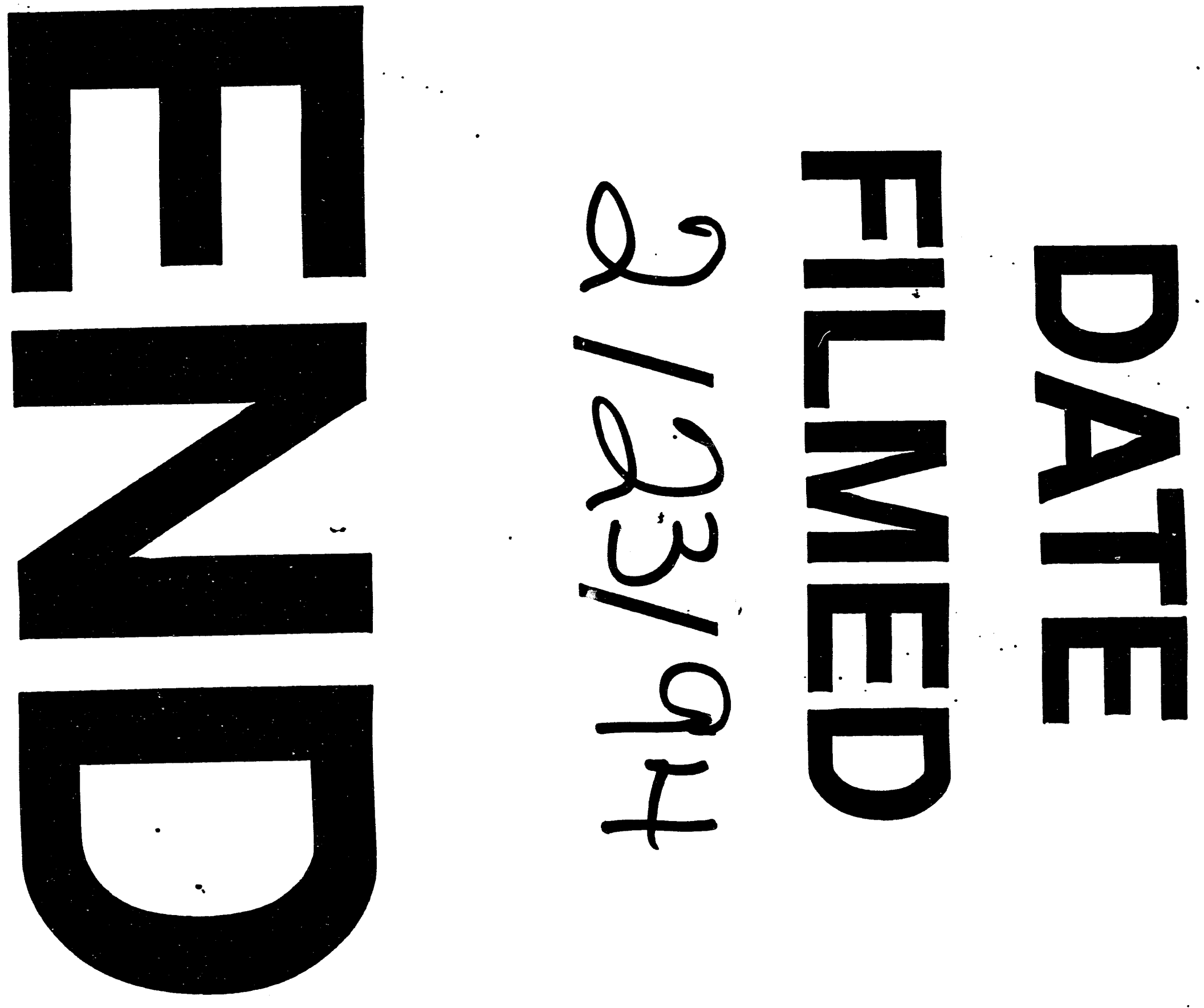
